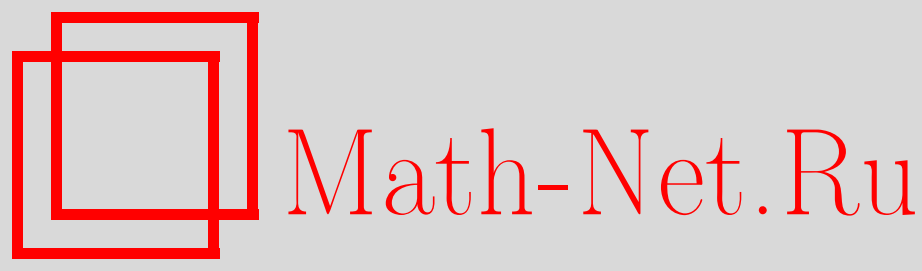

Хуэй-Лин Ли, Жун Линь, Тэн-Цзяо Чжоу, Тао Хуан, Излучение Хокинга как туннелирование с гравитационной обратной связью для черной дыры Керра-анти де Ситтера, ТМФ, 2009, том 161, номер 1, 136-144

DOI: https://doi.org/10.4213/tmf6426

Использование Общероссийского математического портала Math-Net.Ru подразумевает, что вы прочитали и согласны с пользовательским соглашением http://www.mathnet.ru/rus/agreement

Параметры загрузки:

IP : 52.6 .47 .48

26 апреля 2023 г., 18:13:42

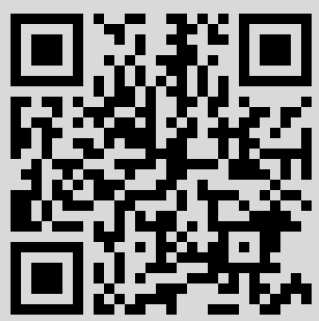




\section{ИЗЛУЧЕНИЕ ХОКИНГА КАК ТУННЕЛИРОВАНИЕ С ГРАВИТАЦИОННОЙ ОБРАТНОЙ СВЯЗЬЮ ДЛЯ ЧЕРНОЙ ДЫРЫ КЕРРА-АНТИ ДЕ СИТТЕРА}

Медвед и Вагенас показали, как можно применить анализ Ангебера-Надалини-Ванзо-Зербини при описании эффектов гравитационного обратного действия для сферически-симметричной черной дыры общего вида. Метод квантового туннелирования Медведа-Вагенаса обобщен на случай стационарной осесимметричной черной дыры Керра в пространственно-временных многообразиях с метрикой анти де Ситтера. Найдены поправки к хокинговскому излучению, вызванные эффектами гравитационной обратной реакции, а также законами сохранения энергии и момента количества движения.

Ключевые слова: ковариантный результат, сохранение энергии, сохранение момента количества движения, обратная связь.

\section{1. ВВЕДЕНИЕ}

С того времени, как Хокинг сделал свое выдающееся открытие, что черная дыра может излучать частицы любого сорта, а ее температура пропорциональна гравитационному полю на ее поверхности (строго говоря, на ее горизонте событий) [1], в литературе были предложены самые разнообразные способы вычисления хокинговского излучения [2]-[10], которые доказывают, что это излучение чисто температурное, что приводит к знаменитому парадоксу потери информации в физике черной дыры [11]. Несмотря на то что настоящее объяснение парадокса потери информации возможно лишь в рамках квантовой теории гравитации или струнной теории, применение квазиклассических методов при исследовании характеристик хокинговского излучения оказалось весьма успешным. Например, можно ожидать, что информация способна выходить наружу, если испускаемое излучение не чисто температурное, но имеет слабые поправки [11]. Интерпретация хокинговского излучения как туннелирования с обратной связью действительно может помочь в разрешении парадокса потери информации, и потому кажется немного странным, что

${ }^{*}$ College of Physics Science and Technology, Shenyang Normal University, Shenyang, China. E-mail: LHL51759@126.com 
лишь в последнее время были предприняты попытки исследования хокинговского излучения как туннелирования с учетом эффектов гравитационного обратного действия.

Исследуя самодействие гравитационного поля и закон сохранения энергии, Парих и Вильчек развили метод квазиклассического туннелирования при вычислении интенсивности излучения черной дыры Шварцшильда. Ими было найдено, что спектр излучения оказывается не чисто температурным, и это открывает возможность существования несущих информацию поправок к излучению [12], [13]. В рамках этой интерпретации туннелирования были найдены туннельные излучения с космологических горизонтов черных дыр в пространствах анти де Ситтера (АдС) и де Ситтера [14], [15]. Далее Жанг и Жао распространили этот метод со случая сферически-симметричных черных дыр на случай аксиально-симметричных черных дыр общего вида [16] и даже на процессы туннелирования массивных и заряженных частиц [17]. С этого момента метод туннелирования применялся многими авторами для исследования хокинговского излучения как туннелирования из различных черных дыр [18]-[25]. Все они отмечали, что спектр излучения отличается от чисто температурного, если принимаются во внимание эффекты обратной связи.

Тем не менее все эти исследования оказываются не вполне удовлетворительными, поскольку ограничиваются изучением метрик срезов постоянного времени в плоском евклидовом пространстве или в пространстве де Ситтера. В рамках этого подхода вводится система координат Пенлеве для того, чтобы устранить сингулярность в системе отсчета. Эти координаты обладают многочисленными достоинствами: метрика регулярна на горизонте событий, в каждой точке пространства в координатах Пенлеве существует времениподобный вектор, после координатного преобразования метрика удовлетворяет условию Ландау синхронизации координатных часов. Тем не менее, хотя система координат Пенлеве и обладает многими преимуществами при изучении эффектов туннелирования для хокинговского излучения, задача построения единой координатной системы Пенлеве оказывается весьма сложной. Более того, при туннелировании через горизонт событий геодезические линии безмассовых частиц должны быть светоподобны, а геодезические линии массивных частиц времениподобны, но это обстоятельство не поддается учету ввиду дуальности де Бройля и в силу самого определения фазовой (групповой) скорости. Наконец, на финальной стадии процесса вычислений требуется интегрировать по энергиям испущенных частиц, что оказывается исключительно трудоемкой процедурой. В этой связи возник новый метод, предложенный Ангебеном, Надалини, Ванзо и Зербини (АНВ3) [26], в рамках которого удается преодолеть все эти трудности и который был применен к хокинговскому излучению. По своей сути ковариантность в методе АНВЗ достигается с помощью применения пространственного собственного расстояния, инвариантного относительно выбора системы координат, что позволяет избежать перехода к координатам Пенлеве и найти уравнение движения излученной 
частицы с помощью уравнения Гамильтона-Якоби. Тем не менее метод АНВЗ ограничивается только ведущим членом и не учитывает эффекты обратной реакции воздействия испущенной частицы на геометрию черной дыры.

Недавно Медведу и Вагенасу удалось обобщить метод АНВЗ на случай квантовой гравитации и дать описание того, как можно учесть эффекты обратной связи [18]. В рамках этого подхода были проведены многочисленные исследования хокинговского излучения как туннелирования из разнообразных сферически-симметричных черных дыр, полученные результаты оказались весьма обнадеживающими [27]-[29]. Но все эти исследования были ограничены случаем черных дыр со сферической симметрией. В одном важном случае, а именно в случае черной дыры Керра-АдС таких исследований сделано не было. Решение типа черной дыры Керра в пространстве АдС получается из уравнений Эйнштейна с отрицательной космологической постоянной. Решение типа черной дыры Керра-АдС переходит в решение типа черной дыры Керра в случае космологической постоянной $\Lambda=0$. Температура черной дыры Керра-АдС минимальна, когда ее радиус оказывается порядка характерного радиуса пространства АдС. Для бо́льших черных дыр Керра-АдС температура, измеряемая по инфракрасному сдвигу на бесконечности, оказывается выше. Отсюда можно предположить, что такие черные дыры могут находиться в стабильном равновесии с термальным излучением при определенной температуре. Метрика Керра-АдС представляет важный пример пространства с метрикой АдС. Пространственно-временные многообразия с метрикой АдС играют важную роль при исследовании основных свойств черных дыр. После того как Хокинг обнаружил свойство потери информации черными дырами, спор относительно природы этого явления продолжался в течение десятилетий без всякого удовлетворительного разрешения. Наконец исследователи пришли к заключению, что информация все-таки сохраняется, если предположить дуальность между струнной теорией в пространстве АдС и конформной теорией поля на границе пространства АдС, находящейся на бесконечности [30] [31].

Благодаря отмеченной выше важной роли черных дыр в метрике АдС в настоящей работе мы предприняли попытку обобщить метод Медведа-Вагенаса на случай черных дыр Керра-АдС. В случае стационарной черной дыры следует учесть не только закон сохранения энергии, но и закон сохранения момента количества движения. Дополнительно, в силу наличия эффекта захвата системы отсчета (frame dragging) координатной системы в пространстве-времени с постоянной скоростью вращения, правильная физическая картина должна быть описана в захваченной системе координат. В настоящей работе мы находим интенсивность туннелирования из черной дыры, учитывая эффекты обратной реакции и законы сохранения энергии и момента количества движения. Результат показывает, что спектр излучения на горизонте событий оказывается не чисто температурным, а интенсивность туннелирования связана с энтропией Бекенштейна-Хокинга и удовлетворяет условиям унитарности. 


\section{2. УРАВНЕНИЯ ГАМИЛЬТОНА-ЯКОБИ И КОВАРИАНТНЫЙ РЕЗУЛЬТАТ}

В гравитации Максвелла-АдС в размерности $(3+1)$ с отрицательной космологической постоянной $\Lambda=-3 / l^{2}$ выражение для метрики черной дыры Керра-АдС имеет вид [32]

$d s^{2}=-\frac{\rho^{2} \Delta_{r} \Delta_{\theta}}{\Sigma^{2}} d t^{2}+\frac{\rho^{2}}{\Delta_{r}} d r^{2}+\frac{\rho^{2}}{\Delta_{\theta}} d \theta^{2}+\frac{\Sigma^{2} \sin ^{2} \theta}{\rho^{2} \Xi^{2}}\left\{d \varphi-\frac{a\left[\Delta_{\theta}\left(r^{2}+a^{2}\right)-\Delta_{r}\right] \Xi}{\Sigma^{2}} d t\right\}^{2}$,

где

$$
\begin{gathered}
\Xi=1-\frac{a^{2}}{l^{2}}, \quad \rho^{2}=r^{2}+a^{2} \cos ^{2} \theta, \quad \Delta_{r}=\left(r^{2}+a^{2}\right)\left(1+\frac{r^{2}}{l^{2}}\right)-2 m r, \\
\Sigma^{2}=\Delta_{\theta}\left(r^{2}+a^{2}\right)^{2}-\Delta_{r} a^{2} \sin ^{2} \theta, \quad \Delta_{\theta}=1-\frac{a^{2}}{l^{2}} \cos ^{2} \theta .
\end{gathered}
$$

Горизонт событий $r=r_{\mathrm{h}}$ удовлетворяет уравнению

$$
\Delta_{r}=\left(r^{2}+a^{2}\right)\left(1+\frac{r^{2}}{l^{2}}\right)-2 m r=0,
$$

причем $r_{\mathrm{h}}$ - наибольший корень данного квадратного уравнения. Площадь горизонта событий равна

$$
A=\frac{4 \pi\left(r_{\mathrm{h}}^{2}+a^{2}\right)}{\Xi}
$$

а выражение для энтропии Бекенштейна-Хокинга имеет вид

$$
S_{\mathrm{BH}}=\frac{A}{4} .
$$

В силу наличия эффекта захвата системы координат в стационарно вращающихся пространственно-временных системах отсчета поле материи на эргосфере вблизи горизонта событий должно также захватываться гравитационным полем с азимутальной угловой скоростью, так что правильная физическая картина должна описываться в захваченной системе координат. Переходя в эту систему координат, имеем уравнение

$$
\Omega=\dot{\varphi}=-\frac{g_{03}}{g_{33}}=\frac{a \Xi\left[\Delta_{\theta}\left(a^{2}+r^{2}\right)-\Delta_{r}\right]}{\Sigma^{2}},
$$

подставляя его в метрику (1), получим

$$
d s^{2}=-\frac{\rho^{2} \Delta_{r} \Delta_{\theta}}{\Sigma^{2}} d t^{2}+\frac{\rho^{2}}{\Delta_{r}} d r^{2}+\frac{\rho^{2}}{\Delta_{\theta}} d \theta^{2} .
$$

В рамках квазиклассического приближения действие на указанном фоне черной дыры удовлетворяет релятивистскому уравнению Гамильтона-Якоби

$$
g^{\mu \nu} \partial_{\mu} I \partial_{\nu} I+\mu^{2}=0,
$$


где $\mu$ - масса туннелирующей частицы. В силу симметрии метрики

$$
I=-\varepsilon t+W(r, \theta)+j \varphi
$$

где $\varepsilon$ - энергия туннелирующей частицы, а $j$ - составляющая обобщенного импульса относительно угловой переменной $\varphi$. Подставляя уравнения (7) и (9) в выражение (8), получим

$$
-\frac{\Sigma^{2}}{\rho^{2} \Delta_{r} \Delta_{\theta}}(\varepsilon-j \Omega)^{2}+\frac{\Delta_{r}}{\rho^{2}}\left(\frac{\partial W(r, \theta)}{\partial r}\right)^{2}+\frac{\Delta_{\theta}}{\rho^{2}}\left(\frac{\partial W(r, \theta)}{\partial \theta}\right)^{2}+\mu^{2}=0 .
$$

Поскольку ведущий вклад происходит от горизонта событий, можно рассмотреть разложение вблизи этого горизонта, что дает

$$
-\frac{\left(a^{2}+r_{\mathrm{h}}^{2}\right)^{2}}{\rho_{\mathrm{h}}^{2} \Delta_{r}^{\prime}\left(r-r_{\mathrm{h}}\right)}\left(\varepsilon-j \Omega_{\mathrm{h}}\right)^{2}+\frac{\Delta_{r}^{\prime}\left(r-r_{\mathrm{h}}\right)}{\rho^{2}}\left(\frac{\partial W(r, \theta)}{\partial r}\right)^{2}+\frac{\Delta_{\theta}}{\rho_{\mathrm{h}}^{2}}\left(\frac{\partial W(r, \theta)}{\partial \theta}\right)^{2}+\mu^{2}=0
$$

где

$$
\begin{array}{cc}
\Delta_{r}=\Delta_{r}^{\prime}\left(r_{\mathrm{h}}\right)\left(r-r_{\mathrm{h}}\right), & \Delta_{r}^{\prime}=\Delta_{r}^{\prime}\left(r_{\mathrm{h}}\right)=\left.\frac{d \Delta_{r}}{d r}\right|_{r=r_{\mathrm{h}}}, \\
\Omega_{\mathrm{h}}=\frac{a \Xi}{a^{2}+r_{\mathrm{h}}^{2}}, & \rho_{\mathrm{h}}^{2}=r_{\mathrm{h}}^{2}+a^{2} \cos ^{2} \theta .
\end{array}
$$

В силу уравнений (9) и (11) классическое действие имеет вид

$$
I=-\varepsilon t+\int \frac{a^{2}+r_{\mathrm{h}}^{2}}{\Delta_{r}^{\prime}\left(r-r_{\mathrm{h}}\right)} \sqrt{\left(\varepsilon-j \Omega_{\mathrm{h}}\right)^{2}-\frac{\rho_{\mathrm{h}}^{2} \Delta_{r}^{\prime}\left(r-r_{\mathrm{h}}\right)}{\left(a^{2}+r_{\mathrm{h}}^{2}\right)^{2}}\left[\frac{\Delta_{\theta}}{\rho_{\mathrm{h}}^{2}}\left(\frac{\partial W(r, \theta)}{\partial \theta}\right)^{2}+\mu^{2}\right]} d r+j \varphi .
$$

Для того чтобы получить верный результат, необходимо использовать собственное пространственное расстояние. Как было показано в работе [26], ввиду потери ковариантности интеграл оказывается неинвариантным в искривленном пространствевремени. Поэтому в методе АНВЗ вводится [26] собственное пространственное расстояние - инвариант координат, задаваемый пространственной метрикой

$$
d \sigma^{2}=\frac{\rho^{2}}{\Delta_{r}} d r^{2}+\frac{\rho^{2}}{\Delta_{\theta}} d \theta^{2}=\frac{\rho_{\mathrm{h}}^{2}}{\Delta_{r}^{\prime}\left(r-r_{\mathrm{h}}\right)} d r^{2}+\frac{\rho_{\mathrm{h}}^{2}}{\Delta_{\theta}} d \theta^{2}
$$

В дальнейшем мы ограничимся вкладом s-волн, т.е. только радиальная составляющая движения частицы будет ненулевой, $d \theta=0$. Поскольку процесс туннелирования происходит в окрестности горизонта событий и ведущий вклад поступает с горизонта, применим разложение вблизи горизонта, что дает

$$
\sigma=\int \sqrt{\frac{\rho_{\mathrm{h}}^{2}}{\Delta_{r}^{\prime}\left(r-r_{\mathrm{h}}\right)}} d r=\frac{2}{\sqrt{\Delta_{r}^{\prime}}} \sqrt{\rho_{\mathrm{h}}^{2}\left(r-r_{\mathrm{h}}\right)},
$$

и мы получаем инвариантный результат

$$
W(\sigma)=\frac{2\left(a^{2}+r_{\mathrm{h}}^{2}\right)}{\Delta_{r}^{\prime}} \int \frac{d \sigma}{\sigma} \sqrt{\left(\varepsilon-j \Omega_{\mathrm{h}}\right)^{2}-\frac{\sigma^{2}}{4}\left(\frac{\Delta_{r}^{\prime}}{a^{2}+r_{\mathrm{h}}^{2}}\right)^{2}\left[\frac{\Delta_{\theta}}{\rho_{\mathrm{h}}^{2}}\left(\frac{\partial W(r, \theta)}{\partial \theta}\right)^{2}+\mu^{2}\right]}
$$


Данный интеграл расходится при $\sigma=0$, что соответствует горизонту. Вычислим его с малой отрицательной добавкой, физическое обоснование которой состоит в том, что рассматривается процесс вне массовой оболочки [26]. Фейнмановский пропагатор в этой картине имеет вид

$$
\frac{1}{\sigma} \rightarrow \frac{1}{\sigma-i 0}=P P \frac{1}{\sigma}+i \pi \delta(\sigma)
$$

Мы получаем таким образом мнимую составляющую, физический смысл которой связан с учетом хокинговского излучения как процесса туннелирования. В итоге имеем ненулевую мнимую составляющую в действии:

$$
I=\frac{2 \pi i\left(r_{\mathrm{h}}^{2}+a^{2}\right)}{\Delta_{r}^{\prime}}\left(\varepsilon-j \Omega_{\mathrm{h}}\right)+\text { вещественный вклад. }
$$

\section{3. ИЗЛУЧЕНИЕ ХОКИНГА \\ КАК ТУННЕЛИРОВАНИЕ С ОБРАТНОЙ СВЯЗЬЮ}

Чтобы изучить хокинговское излучение как туннелирование с обратной реакцией, обратимся к закону сохранения массы для черной дыры. В пространстве АдС было найдено выражение для энергии $E$ и момента количества движения $J$ [33]:

$$
E=\frac{m}{\Xi}, \quad J=\frac{a m}{\Xi^{2}} .
$$

Когда частица с массой $\omega$ испускается с горизонта событий, энергия и момент количества движения частицы задаются выражениями $\omega / \Xi$ и $a \omega / \Xi^{2}$ соответственно. Для удобства перепишем уравнение (18) в виде

$$
\begin{aligned}
I & =\frac{2 \pi i\left(r_{\mathrm{h}}^{2}+a^{2}\right)}{\Delta_{r}^{\prime}\left(r_{\mathrm{h}}\right)}\left(\frac{\omega}{\Xi}-\frac{a \omega}{\Xi^{2}} \Omega_{\mathrm{h}}\right)+\text { вещественный вклад }= \\
& =\frac{2 \pi i r_{\mathrm{h}}^{2}}{\Delta_{r}^{\prime}\left(r_{\mathrm{h}}\right)} \frac{\omega}{\Xi}+\text { вещественный вклад. }
\end{aligned}
$$

Учитывая эффекты обратной реакции для черной дыры Керра-АдС, мы должны модифицировать уравнение (20). Чтобы обеспечить сохранение энергии и момента количества движения, зафиксируем полную массу и полный момент количества движения пространства-времени, но позволим изменяться массе и моменту количества движения черной дыры. Когда частица с массой $\omega$ и моментом количества движения $j$ испускается с горизонта событий, энергия и момент количества движения черной дыры становятся равными $E-\varepsilon=(m-\omega) / \Xi$ и $J-j=a(m-\omega) / \Xi^{2}$. Кроме того, как было отмечено Медведом и Вагенасом, ввиду присутствия квантовой неопределенности и квантовой смазанности переход должен происходить "постепенно" [18], поэтому следует заменить $r_{\mathrm{h}}(m)$ на $r_{\mathrm{h}}^{\prime}(m-\omega)$, а потом проинтегрировать по переменной $\omega$ с подходящей мерой. Перепишем мнимую составляющую действия в более сжатом виде:

$$
\operatorname{Im} I=\left.\operatorname{Im} \int_{0}^{\varepsilon} I\right|_{m \rightarrow m-\omega} d \frac{\omega^{\prime}}{\Xi}=-\frac{2 \pi}{\Xi} \int_{m}^{m-\omega} \frac{\left(r_{\mathrm{h}}^{\prime}\right)^{2}}{\Delta_{r}^{\prime}\left(r_{\mathrm{h}}^{\prime}\right)} d\left(m-\omega^{\prime}\right),
$$


где

$$
\Delta_{r}^{\prime}\left(r_{\mathrm{h}}^{\prime}\right)=\left.\frac{d \Delta_{r}}{d r}\right|_{r=r_{\mathrm{h}}^{\prime}}=2 r_{\mathrm{h}}^{\prime}\left[\frac{1}{2}\left(1+\frac{a^{2}}{l^{2}}\right)-\frac{a^{2}}{2\left(r_{\mathrm{h}}^{\prime}\right)^{2}}+\frac{3\left(r_{\mathrm{h}}^{\prime}\right)^{2}}{2 l^{2}}\right] .
$$

Из уравнения для горизонта событий $\Delta_{r}^{\prime}\left(r_{\mathrm{h}}^{\prime}\right)=0$ имеем

$$
m-\omega^{\prime}=\frac{1}{2 r_{\mathrm{h}}^{\prime}}\left[\left(\left(r_{\mathrm{h}}^{\prime}\right)^{2}+a^{2}\right)\left(1+\frac{\left(r_{\mathrm{h}}^{\prime}\right)^{2}}{l^{2}}\right)\right],
$$

так что

$$
d\left(m-\omega^{\prime}\right)=\left[\frac{1}{2}\left(1+\frac{a^{2}}{l^{2}}\right)-\frac{a^{2}}{2\left(r_{\mathrm{h}}^{\prime}\right)^{2}}-\frac{3\left(r_{\mathrm{h}}^{\prime}\right)^{2}}{2 l^{2}}\right] d r_{\mathrm{h}}^{\prime}
$$

Тем самым получим следующее выражение:

$$
\operatorname{Im} I=-\frac{2 \pi}{\Xi} \int_{r_{\mathrm{i}}(m)}^{r_{\mathrm{f}}(m-\omega)} \frac{r_{\mathrm{h}}^{\prime}}{2} d r_{\mathrm{h}}^{\prime}=-\frac{2 \pi}{\Xi}\left(r_{\mathrm{f}}^{2}-r_{\mathrm{i}}^{2}\right),
$$

где $r_{\mathrm{i}}=r_{\mathrm{h}}(m)$ - первоначальный радиус черной дыры, а $r_{\mathrm{f}}=r_{\mathrm{h}}^{\prime}(m-\omega)-$ конечный радиус после завершения процесса туннелирования частицы с массой $\omega$. В результате интенсивность туннелирования с учетом квантовой поправки имеет вид

$$
\Gamma \sim e^{-2 \operatorname{Im} I}=\exp \left(\frac{\pi}{\Xi}\left(r_{\mathrm{f}}^{2}-r_{\mathrm{i}}^{2}\right)\right)=\exp \left(\frac{\pi\left(r_{\mathrm{f}}^{2}+a^{2}\right)}{\Xi}-\frac{\pi\left(r_{\mathrm{i}}^{2}+a^{2}\right)}{\Xi}\right)=e^{\Delta S_{\mathrm{BH}}},
$$

где $\Delta S_{\mathrm{BH}}=S(m-\omega)-S(m)-$ изменение энтропии Бекенштейна-Хокинга, обусловленное испусканием частицы с массой $\omega$ из черной дыры. Уравнение (26) наглядно показывает, что интенсивность туннелирования под горизонтом событий связана с изменением энтропии Бекенштейна-Хокинга, а спектр энергий излучения оказывается не чисто температурным.

\section{4. ЗАКЛЮЧЕНИЕ}

Мы обобщили метод квантового туннелирования Медведа и Вагенаса на случай стационарной аксиально-симметричной черной дыры Керра в пространстве-времени АдС, примененяя метод АНВЗ и учитывая эффекты обратной реакции. Следует отметить два существенных момента: с одной стороны, принимался в расчет не только закон сохранения энергии, но и закон сохранения момента количества движения, с другой стороны, рассмотрение велось в захваченной системе координат в силу наличия эффекта захвата системы отсчета в пространстве-времени с постоянной скоростью вращения. Заметим, что при нашем подходе ковариантное описание сингулярности на горизонте событий происходит без перехода в систему координат Пенлеве. Кроме того, предлагаемый подход сравнительно прост, так как в нем не рассматриваются геодезические линии и не применяется комплексное интегрирование. Тем не менее ответ, полученный в рамках этого подхода, совпадает с ответом, полученным в рамках метода туннелирования Париха-Вильчека. Результат показывает, что фактический спектр излучения отклоняется от чисто температурного 
спектра и находится в согласии с лежащей в основе унитарной теорией; иными словами, можно добиться сохранения информации, если принять в расчет эффекты обратной реакции.

Добавим, что можно распространить метод туннелирования на класс динамических черных дыр [34]; в этом случае туннелирование происходит под горизонтом захвата. В стационарных черных дырах горизонт захвата совпадает с горизонтом событий, но в нестационарном случае в силу процесса испарения эти два горизонта не совпадают. Если произвести новое координатное преобразование, то можно исследовать туннелирование под горизонтом событий [35]. Для сферически-симметричных черных дыр подход настоящей работы был непосредственно применен к горизонтам в случае отсутствия сингулярностей в класификации Бронникова и соавторов [36]. Но если сингулярность имеет место, то применимость предлагаемого подхода остается открытым вопросом.

Благодарности. Авторы признательны рецензенту за ценные предложения, учет которых существенно улучшил содержание статьи. Работа была финансово поддержана Национальным фондом научных исследований КНР (грант № 10773008).

\section{Список литературы}

[1] S. W. Hawking, Comm. Math. Phys., 43:3 (1975), 199-220; Nature, 248 (1974), 30-31.

[2] T. Damour, R. Ruffini, Phys. Rev. D, 14:2 (1976), 332-334.

[3] Z. H. Li, Z. Zhao, Acta Phys. Sinica, 46:7 (1997), 1273-1281.

[4] Q.-Q. Jiang, S.-Q. Wu, Phys. Lett. B, 647:2-3 (2007), 200-206; Q.-Q. Jiang, S.-Q. Wu, X. Cai, Phys. Rev. D, 75:6 (2007), 064029.

[5] U. Khanal, Phys. Rev. D, 28 (1983), 1291; U. Khanal, N. Panchapakesan, Phys. Rev. D, 24:4 (1981), 829-834.

[6] Z. Zhao, J.Y. Zhu, Acta Phys. Sinica, 48:8 (1999), 1558-1564; Z. Zhao, C. Q. Yang, Q. A. Ren, Gen. Relativ. Grav., 26:11 (1992), 1055-1065; Z. Zhao, X.X. Dai, Modern Phys. Lett. A, 7:20 (1992), 1771-1778.

[7] S. Sannan, Gen. Relativ. Grav., 20:3 (1988), 239-246.

[8] E. Keski-Vakkuri, P. Kraus, Phys. Rev. D, 54:12 (1996), 7407-7420.

[9] S. Shankaranarayanan, T. Padmanabhan, K. Srinivasan, Class. Quant. Grav., 19:10 (2002), 2671-2687.

[10] L. Vanzo, Phys. Rev. D, 55:4 (1997), 2192-2201.

[11] S. W. Hawking, Phys. Rev. D, 14:10 (1976), 2460-2473.

[12] M. K. Parikh, F. Wilczek, Phys. Rev. Lett., 85:24 (2000), 5042-5045; M. K. Parikh, Internat. J. Modern Phys. D, 13:10 (2004), 2351-2354; Phys. Lett. B, 546:3-4 (2002), 189-195.

[13] P. Kraus, F. Wilczek, Nucl. Phys. B, 437:1 (1995), 231-242.

[14] A. J. M. Medved, Phys. Rev. D, 66:12 (2002), 124009.

[15] S. Hemming, E. Keski-Vakkuri, Phys. Rev. D, 64:4 (2001), 44006.

[16] J. Y. Zhang, Z. Zhao, Phys. Lett. B, 618:1-4 (2005), 14-22; Modern Phys. Lett. A, 20:22 (2005), 1673-1681.

[17] J. Y. Zhang, Z. Zhao, JHEP, 10 (2005), 10055; Nucl. Phys. B, 725:1-2 (2005), 173-180.

[18] A. J. M. Medved, E. C. Vagenas, Modern Phys. Lett. A, 20:23 (2005), 1723-1728; 20:32 (2005), 2449-2453.

[19] M. Arzano, A. J.M. Medved, E. C. Vagenas, JHEP, 09 (2005), 037; arXiv: hep-th/0505266. 
[20] M. R. Setare, E. C. Vagenas, Internat. J. Modern Phys. A, 20:30 (2005), 7219-7232; M. Arzano, Modern Phys. Lett. A, 21:1 (2006), 41-48; arXiv: hep-th/0504188.

[21] H.-L. Li, S.-Z. Yang, Q.-Q. Jiang, D.-J. Qi, Phys. Lett. B, 641:2 (2006), 139-144; H.-L. Li, D.-J. Qi, S.-Z. Yang, Gen. Relativ. Grav., 39:2 (2007), 179-188.

[22] Q.-Q. Jiang, S.-Q. Wu, X. Cai, Phys. Rev. D, 73:6 (2006), 064003.

[23] G.-Q. Li, Europhys. Lett., 76:2 (2006), 203-207; Гу-Цян Ли, ТМФ, 151:1 (2007), 172-175; 153:3 (2007), 358-362; 149:1 (2006), 60-64.

[24] E. C. Vagenas, Phys. Lett. B, 503:3-4 (2001), 399-403; 533:3-4 (2002), 302-306; Modern Phys. Lett. A, 17:10 (2002), 609-618.

[25] S.-Z. Yang, Chinese Phys. Lett., 22:10 (2005), 2492-2495; S.-Z. Yang, Q.-Q. Jiang, H.-L. Li, Chinese Phys., 14:12 (2005), 2411-2414.

[26] M. Angheben, M. Nadalini, L. Vanzo, S. Zerbini, JHEP, 05 (2005), 014.

[27] Q. Q. Jiang, S. Q. Wu, X. Cai, Acta. Phys. Sinica, 56:6 (2007), 3083-3087.

[28] H.-L. Li, S.-Z. Yang, Europhys. Lett., 79:2 (2007), 20001.

[29] S.-Z. Yang, Q.-Q. Jiang, Internat. J. Theoret. Phys., 46:8 (2007), 2138-2145.

[30] J. M. Maldacena, Adv. Theor. Math. Phys., 2:2 (1998), 231-252.

[31] S. S. Gubser, I. R. Klebanov, A. M. Polyakov, Phys. Lett. B, 428:1-2 (1998), 105-114.

[32] B. Carter, Comm. Math. Phys., 10 (1968), 280-310.

[33] S. Das, R. B. Mann, JHEP, 08 (2000), 033; arXiv: hep-th/0008028.

[34] R. Di Criscienzo, M. Nadalini, L. Vanzo, S. Zerbini G. Zoccatelli, Phys. Lett. B, 657:1-3 (2007), 107-111; arXiv:0707.4425.

[35] H.-L. Li, S.-Z. Yang, T.-J. Zhou, R. Lin, Europhys. Lett., 84:2 (2008), 20003.

[36] K. A. Bronnikov, E. Elizalde, S. D. Odintsov, O. B. Zaslavskii, Phys. Rev. D, 78:6 (2008), 064049; arXiv: 0805.1095.

Поступила в редакцию 30.06.2008, после доработки 20.10.2008 